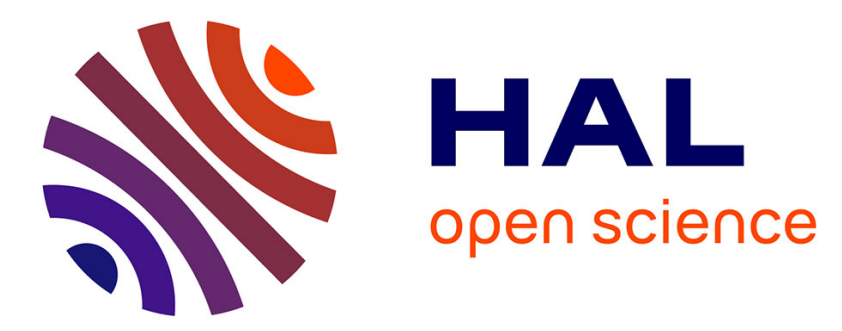

\title{
On the localization of shallow water waves by a random bottom
}

\author{
E. Guazzelli, E. Guyon, B. Souillard
}

\section{To cite this version:}

E. Guazzelli, E. Guyon, B. Souillard. On the localization of shallow water waves by a random bottom. Journal de Physique Lettres, 1983, 44 (20), pp.837-841. 10.1051/jphyslet:019830044020083700 . jpa00232271

\section{HAL Id: jpa-00232271 https://hal.science/jpa-00232271}

Submitted on 1 Jan 1983

HAL is a multi-disciplinary open access archive for the deposit and dissemination of scientific research documents, whether they are published or not. The documents may come from teaching and research institutions in France or abroad, or from public or private research centers.
L'archive ouverte pluridisciplinaire HAL, est destinée au dépôt et à la diffusion de documents scientifiques de niveau recherche, publiés ou non, émanant des établissements d'enseignement et de recherche français ou étrangers, des laboratoires publics ou privés. 


\title{
LE JOURNAL DE PHYSIQUE-LETTRES
}

J. Physique - LETTRES 44 (1983) L-837 - L-841

15 OCTOBRE 1983, PAGE L- 837

Classification

Physics Abstracts

$47.35-71.30-71.50$

\section{On the localization of shallow water waves by a random bottom}

\author{
E. Guazzelli (*), E. Guyon
}

Laboratoire d'Hydrodynamique et de Mécanique Physique, ESPCI, 10, rue Vauquelin, 75231 Paris Cedex 05, France

\author{
and B. Souillard \\ Centre de Physique Théorique, Ecole Polytechnique, 91128 Palaiseau Cedex, France
}

(Reçu le 30 mai 1983, accepté le 30 août 1983)

\begin{abstract}
Résumé. - La vitesse de propagation d'une onde en eau peu profonde varie avec sa profondeur $h(x)$. On s'attend à ce qu'une onde plane de vecteur d'onde $\mathbf{k}$ parallèle à $x$ soit partiellement réfléchie par les modulations du fond. Nous prédisons que ceci doit conduire, dans le cas d'une modulation aléatoire du fond sur une distance assez longue, à une localisation exponentielle de tous les modes propres et à une réflexion totale d'une onde incidente, phénomène analogue à celui de la localisation, découvert et largement étudié en physique des solides. Une première étape de l'étude expérimentale de la localisation considère l'effet d'une modulation spatialement périodique du fond (réflexion de Bragg) et l'effet supplémentaire d'une variation aléatoire de l'amplitude de cette modulation.

Abstract. - Linear shallow water waves have a velocity which varies with the water depth $h(x)$. We expect that a plane wave (wave vector $\mathbf{k}$ parallel to $x$ ) will be partially reflected by modulations of the bottom. We argue that this should lead, in the presence of a random modulation of the depth extending over a long enough distance, to exponential localization of all proper modes and to the total reflection of an incident wave, a phenumenon analogous to the one of localization discovered and widely studied in solid state physics. As a first step in the experimental study of localization of water waves, we show the effect of a periodic modulation of the bottom (Bragg scattering) and the additional effect created by using a random amplitude.
\end{abstract}

The study of surface waves over a rough bottom is a subject of importance to coastal engineers and sedimentologists. In the simple approximation where the bottom pattern is independent of the wave formation (which excludes more complex cases, due to redistribution of thin sediments

$\left({ }^{*}\right)$ Present address : Département de Physique des Systèmes Désordonnés, Université de Provence, 13397 Marseille Cedex 13. 
by the flow), the,velocity of linear surface waves is a function of the depth $h(\mathbf{r})$ in the limit of a wavelength $\lambda$ large with respect to $h$. Obstacles as well as undulations of the bed cause partial reflection of incident waves. The subject is a well documented one from both the theoretical and experimental sides. The recent work of Davies [1] is an example of the first type. Making use of a Fourier analysis of a rough surface and using the principle of superposition of linear equations, Davies reduces the problem to that of a single sinusoidal period of a strutcture with wave vector, $\mathbf{k}$, parallel to that of the modulation, $\mathbf{K}$. One of the conclusions of this study is the existence of a strong reflection coefficient when $2 k / K=1$. This result is the equivalent of the Bragg condition in one-dimensional models of solid state physics which causes the existence of a gap for similar Brillouin zone conditions in the case of small periodic potentials. The energy splitting would be, in the present case, the difference in energy between waves having their nodes centred at the crests of the modulation or on the valleys. Recent experiments by Heathershaw [2] with plane waves in a long narrow channel and a sinusoidal bottom tend to confirm this analysis.

Using a perturbation expansion, Davies [1] was able to treat the more general case of a nonsinusoidal periodic modulation of small amplitude which he analysed by Fourier series. There is, however, a crucial step in the extension of such results from periodic or nearly periodic bottoms to truly random ones. It is well known in solid state physics that the propagation of Bloch waves in a random potential is dramatically different from that in a periodic one : localization of the stationary waves can result from disorder. In the particular case of one-dimensional systems, such as a metallic wire with very small cross section, a surprising prediction of the theory was that the system should be an insulator at $T=0$ whatever the nature and the strength of the disor$\operatorname{der}[3,4]$.

It is interesting to see whether, despite the difference in the nature, scale and equations for the two problems, localization concepts can predict new phenomena in the physics of rough bottom surface waves. On the other hand, if such phenomena are observable, hydrodynamics would provide a beautiful macroscopic model, in one and two dimensions, of a phenomenon theoretically understood but whose manifestations are only indirect because of its microscopic character.

In the one-dimensional case, the linear equation of shallow water waves [5] is

$$
\rho g \frac{\partial}{\partial x}\left[h(x) \frac{\partial}{\partial x} u(x, t)\right]=\rho \frac{\partial^{2} u(x, t)}{\partial t^{2}}
$$

where $g$ is the acceleration due to gravity, $\rho$ the density of the liquid, $u(x, t)$ is the (small) amplitude of the wave at time $t$ at point $x$ [5]. The corresponding stationary equation at frequency $\omega$ reads :

$$
\rho g \frac{\partial}{\partial x}\left[h(x) \frac{\partial}{\partial x} u_{\omega}(x)\right]=-\rho \omega^{2} u_{\omega}(x) \text {. }
$$

In the case of a horizontally flat bottom, equation 1 reduces to

$$
g h \frac{\partial^{2}}{\partial x^{2}} u(x, t)=\frac{\partial^{2} u(x, t)}{\partial t^{2}}
$$

whose stationary solutions are plane waves with velocity

$$
c= \pm \sqrt{g h} .
$$

In the case of a periodic bottom, of period $\Lambda$, there appear forbidden bands, and for frequencies in these bands, there are no stationary states, and initial states built up with such frequencies would be completely reflected, whereas for frequencies in the pass bands, the stationary states are of the kind $u_{\varphi}(x) \mathrm{e}^{i \varphi x}$ where $u_{\varphi}(x)$ is a periodic function of period $\Lambda$. Initial states with frequencies in a pass band would propagate to infinity as $t \rightarrow \infty$. 
In contrast, if we apply the concepts of localization theory to(1) with a random bottom potential, we predict that all stationary physical solutions of (1) should be exponientially decaying; a weaker consequence is that if one sends in the system some wave it will be completely reflected; in other words, the transmitted wave should decay exponentially with distance $x$ over a disordered bottom. We can make these conclusions safely using what is known from localization theory [3, 4]. However, in contrast to the Schrödinger equation or finite difference tight-binding models which are usually studied, the rate of exponential decay $\gamma(\omega)$ at frequency $\omega$ will tend to 0 with the frequency; in other words, the localization length $\xi(\omega)$ defined as $\gamma(\omega)^{-1}$, diverges for $\omega \rightarrow 0$. In fact equations 1 and $1^{\prime}$ are the continuum analogs of the equations describing phonons in condensed matter physics, and the previous remark is a consequence of the difference from an electron propagation equation.

The basic mechanism responsible for localization is the destructive phase interference between the incident wave and the various reflected waves from various obstacles. This is a large scale effect which should not depend on a short-range cut-off, so that we can use the qualitative predictions obtained from the discrete analog of $\left(1^{\prime}\right)$.

We have started an experimental program to test these predictions and to evaluate localization effects in parallel with numerical simulations. As a first step in the experiment, we considered the effect of a sinusoidal bed of limited length

$$
y_{b}(x)=b \sin (K x+\delta), \quad L_{1} \leqslant x \leqslant L_{2},
$$

in an otherwise flat bottom $y_{b}(x)=0$ [6]. Subsequently, we consider the effect of using random values of $b$ for the different periods of the modulations (Fig. 1).

We use a channel having the following geometry : length : $1.5 \mathrm{~m}$; width $: 0.35 \mathrm{~m}$. The average water depth $h$ is varied between 1 and $3 \mathrm{~cm}$. A vertical wave generator creates a plane wave of amplitude $a(x)$ :

$$
y_{\mathrm{s}}=a(x) \cos (k x-\omega t)
$$

at one end of the water tank, with an absorbing beach with a $12^{\circ}$ slope at the opposite one. In the range of wavelengths used $(2 \pi / k=5$ to $20 \mathrm{~cm})$ and using waves of small amplitude $a(\sim h / 10)$, we found equation 3 to apply in the case of flat bottom as expected from the shallow water wave conditions [5].

For a given wavelength and amplitude of the wave, we define the transmission coefficient $T(\omega)$ as the ratio of the amplitude $a$ of the wave for a given depth (or average depth) measured next to the beach for a flat and a modulated bottom. $T$ is simply measured from the ratio, in the two cases, of the amplitude of the deflection of a strobe beam obliquely incident across the water depth. Figure $2 a$ is obtained for a sinusoidal bottom with $m\left(=K\left(L_{2}-L_{1}\right) / 2\right)=16$ periods and amplitude $b=5 \mathrm{~mm}$. It has a sharp transmission minimum at a frequency $\omega=5.5 \mathrm{~Hz}$ such that the Bragg condition $\lambda=2 \Lambda=10 \mathrm{~cm}$ is fulfilled $(\Lambda=2 \pi / K$ is the period of the bottom [7].) Due to the finite number of bumps we expect secondary minima in wave vector separated by $|\delta k| / K=1 / 2 m$ as predicted by Davies ([6], Fig. 2). Only the first two secondary minima have been observed.

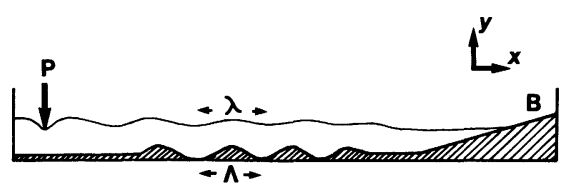

Fig. 1. - Schematic of the experiment. The observation of the amplitude of the wave uses the deflection of an oblique light beam across the water depth. P : vertical plunger ; B : absorbing beach with $12^{\circ}$ slope. 


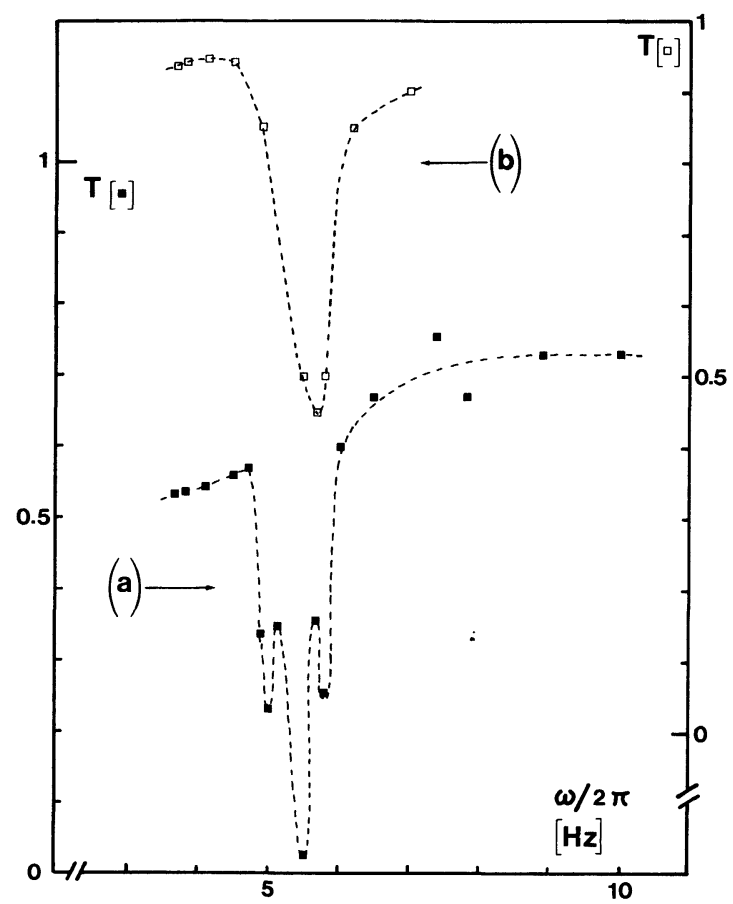

Fig. 2. $-a$ ) The transmission coefficient $T$ is defined as the ratio of the transmitted amplitude for a periodically modulated bottom over that for a flat one having the same average depth $(h=3 \mathrm{~cm})$. The dotted curves are interpolations between the experimental points which are given with a $10 \%$ absolute accuracy. The period of the modulation of the bottom is $\Lambda=5 \mathrm{~cm}$, the amplitude $b=5 \mathrm{~mm}$. $b$ ) Same figure as figure $2 a$ except for the random variation of amplitude $b$ of the crests of the bottom between +5 and $-5 \mathrm{~mm}$.

Figure $2 b$ was obtained with the same wavelength $\Lambda=5 \mathrm{~cm}$ but with the amplitudes $b(x)$ of the $m$ periods drawn at random from +5 to $-5 \mathrm{~mm}$. The transmission coefficient curve has the same dip around the Bragg condition but it is smeared out, thus indicating the effect of randomness on the curve of the transmitted amplitude.

We are developing the experiments on completely random beds, in parallel with a numerical study using equation $1^{\prime}$ to check if localization might be observed in such experiments.

This amounts to asking the order of magnitude of the localization length $\xi(\omega)$ (with respect to the ordinary attenuation length of the wave and to the length of the experimental tank). There are systematic methods to estimate it and practical computations are being carried out. As a matter of principle, it can be said that one can get arbitrarily small localization length $\xi(\omega)$ for $\left(1^{\prime}\right)$ by choosing large disorder, i.e., wilder random fluctuations of the bottom. Clearly if one wants a very shart localization length, one will step out the range of validity of (1) : non-linear phenomena are no longer negligible. However, we think that, here again, the localization phenomenon in the linear regime should have some influence on the nonlinear one. This will be developed in future work.

Another question concerns two-dimensional systems. Adapting the usual localization theory, we predict that, in some range of disorder and frequency, the proper modes of the linear equation will again be exponentially localized.

Besides the random case, another case of interest would be the one of an almost periodic bottom, e.g., $\cos K x+\cos \alpha K x, \alpha$ being irrational. The theory suggests there again many interesting phenomena : in particular forbidden bands could be dense and, in some regimes, stationary modes could be localized. 
In conclusion, we have tried to throw new light on a classical problem in hydrodynamics which, possibly, can provide new practical solutions to oceanographers. In this respect our motivation is very similar to that stressed by Hodges [8] in classical acoustics with random scatterers.

\section{Acknowledgments.}

The experiments have largely benefitted from the training period of A. Tuel, scholar at ESPCI.

We are grateful to A. G. Davies and M. S. Longuet Higgins for information concerning the oceanographic aspects of the problem. The meeting organized in Cambridge by C. J. Hodges in September, 1982, provided a first opportunity of contact on this problem between acousticians and solid state physicists. We also greatly appreciated discussions with C. D. Mitescu, who is presently simulating this problem numerically, and with F. Delyon.

\section{References}

[1] Davies, A. G., J. Mar. Res. 40 (1982) 331.

[2] Heathershaw, A. D., Nature 296 (1982) 343.

[3] Thouless, D. J., Phys. Rep. C 13 (1974) 94.

[4] Thouless, D. J., in Ill-condensed matter, Les Houches (North Holland) 1978.

[5] The conditions of validity of the potential flow approximation leading to this equation are : small amplitude $a$ of the wave and small modulation of the bottom with respect to average depth ; wavelength of the wave $\lambda$ large with respect to the capillarity length $\sqrt{\sigma / \rho g}(\sigma=$ surface tension) and the depth. The problem is introduced in : LAMB, H., Hydrodynamics (Dover Publ., New York) 1945, chap. IX.

Its application to surface wave for variable depth is discussed by : STOKER, J. J., Q. Appl. Math. V (1947) 1.

[6] The problem has been analysed in a linear approximation by DAviEs, A. G., Dyn. Atmos. Oceans 6 (1982) 207.

[7] The analysis in terms of wavelength rather than frequency can be done only by reference to the wave over a flat bottom as is also done in the perturbation approach of Davies. However, as in the case of the dispersion relation for electrons in solid state physics, we expect a gap to appear in frequency, not in wave vector. However, for a finite number of the periods, the gap should manifest itself as a discrete series of frequencies with a poor transmission coefficient.

[8] A similar project has been carried out by Hodges and Woodhouse using an analog model of a stretched string loaded with irregularly spaced masses.

HodGES, C. H., J. Sound Vib. 82 (1982) 411.

Hodges, C. H., Woodhouse, J., to appear in J. Acoust. Soc. Am. 